\title{
The covering number for category and partition relations on $P_{\omega}(\lambda)$
}

\author{
by \\ Pierre Matet (Caen)
}

\begin{abstract}
We show that $\operatorname{cov}(M)$ is the least infinite cardinal $\lambda$ such that $P_{\omega}(\lambda)$ (the set of all finite subsets of $\lambda$ ) fails to satisfy a certain natural generalization of Ramsey's Theorem.
\end{abstract}

0. Introduction. The covering number for category $(\operatorname{cov}(M))$ is known to play an important part in the study of partition properties of ideals on $\omega$. Namely, we have $K^{+} \rightarrow\left(K^{+}\right)^{2}$ for every ideal $K$ on $\omega$ with less than $\operatorname{cov}(M)$ generators (see [14]). On the other hand, there exists an ideal $K$ on $\omega$ generated by $\operatorname{cov}(M)$ sets such that $K^{+} \nrightarrow\left(K^{+}, \omega\right)^{2}$ (see [10]). In the present paper, we investigate the relationship between $\operatorname{cov}(M)$ and partition properties for ideals on $P_{\omega}(\lambda), \lambda$ an infinite cardinal. The partition relation we are mostly interested in, $J^{+} \stackrel{\omega}{\rightarrow}\left(J^{+}\right)^{2}$, is of a mixed type, in the sense that its definition involves functions $F: \omega \times P_{\omega}(\lambda) \rightarrow 2$. We show, as above, that $J^{+} \stackrel{\omega}{\rightarrow}\left(J^{+}\right)^{2}$ for every fine ideal $J$ on $P_{\omega}(\lambda)$ generated by less than $\operatorname{cov}(M)$ sets. In particular, $I_{\omega, \lambda}^{+} \stackrel{\omega}{\rightarrow}\left(I_{\omega, \lambda}^{+}\right)^{2}$ for every $\lambda<\operatorname{cov}(M)$, where $I_{\omega, \lambda}$ denotes the smallest fine ideal on $P_{\omega}(\lambda)$. Observe that for $\lambda=\omega$, $I_{\omega, \lambda}^{+} \stackrel{\omega}{\rightarrow}\left(I_{\omega, \lambda}^{+}\right)^{2}$ is just a reformulation of Ramsey's Theorem [13]. We also show that $I_{\omega, \lambda}^{+} \stackrel{\omega}{\leftrightarrow}\left(I_{\omega, \lambda}^{+}\right)^{2}$ for all $\lambda \geq \operatorname{cov}(M)$. This result emphasizes the heterogeneity of $I_{\omega, \lambda}$, i.e. the fact that the members of $I_{\omega, \lambda}^{+}$are not all alike, since it was shown in [8] that for every $\lambda$, there is a fine ideal $J$ on $P_{\omega}(\lambda)$ such that $J^{+} \stackrel{\omega}{\rightarrow}\left(J^{+}\right)^{2}$.

The paper is organized as follows. The two results mentioned above are to be found in Sections 3 and 4. Section 1 deals with notation and basic

2000 Mathematics Subject Classification: 03E02, 03E17.

Key words and phrases: partition relations, $P_{\omega}(\lambda)$, covering number. 
definitions. It is shown in Section 2 that $I_{\omega, \lambda}$ is a weak $\chi$-point if and only if $\lambda<\operatorname{cov}(M)$. We prove in Section 5 that $\operatorname{cov}(M)=2^{\lambda}$ if and only if every fine ideal $J$ on $P_{\omega}(\lambda)$ with less than $2^{\lambda}$ generators can be extended to a prime ideal $K$ on $P_{\omega}(\lambda)$ such that $K^{+} \stackrel{\omega}{\rightarrow}\left(K^{+}\right)^{2}$. A companion result deals with extension to a prime $\chi$-point. Finally, in Section 6 we derive another remarkable property shared by all fine ideals $J$ on $P_{\omega}(\lambda)$ which are generated by less than $\operatorname{cov}(M)$ sets.

1. Notation. In this section we review some basic definitions.

Given an infinite set $S$, an ideal on $S$ is a collection $J$ of subsets of $S$ such that (i) $\{s\} \in J$ for every $s \in S$, (ii) $P(A) \subseteq J$ for all $A \in J$, (iii) $A \cup B \in J$ whenever $A, B \in J$, and (iv) $S \notin J$.

Let $J$ be an ideal on $S$.

- $\operatorname{cof}(J)$ denotes the least cardinality of any $X \subseteq J$ such that $J=$ $\bigcup_{A \in X} P(A)$.

- $J$ is prime if $J \cap\{A, S-A\} \neq \emptyset$ for all $A \subseteq S$.

- $J^{+}=P(S)-J$ and $J^{*}=\{A \subseteq S: S-A \in J\}$.

- $J \mid A=\{B \subseteq S: B \cap A \in J\}$ for all $A \in J^{+}$.

Let $K$ be an ideal on $\omega$.

- $K$ is weakly selective if given $A \in K^{+}$and $B_{n} \in K$ for $n \in \omega$, there is $C \in K^{+} \cap P(A)$ such that $m \notin B_{n}$ for all $n, m \in C$ with $n<m$.

- $K$ is a $P$-point if given $B_{n} \in K$ for $n \in \omega$, there is $C \in K^{*}$ such that $C \cap B_{n}$ is finite for every $n \in \omega$.

- $K$ is a $Q$-point if given $g: \omega \rightarrow \omega$, there is $A \in K^{*}$ such that $g(n) \leq m$ for all $n, m \in A$ with $n<m$.

- $K^{+} \rightarrow\left(K^{+}\right)^{2}$ asserts that given $A \in K^{+}$and $F: \omega \times \omega \rightarrow 2$, there is $B \in K^{+} \cap P(A)$ such that $F$ is constant on $\{(n, m) \in B \times B: n<m\}$.

- $K^{+} \nrightarrow\left(K^{+}\right)^{2}$ means that $K^{+} \rightarrow\left(K^{+}\right)^{2}$ does not hold.

- For each ordinal $\alpha$ with $2 \leq \alpha \leq \omega, K^{+} \rightarrow\left(K^{+}, \alpha\right)^{2}$ means that given $A \in K^{+}$and $F: \omega \times \omega \rightarrow 2$, there is either $B \in K^{+} \cap P(A)$ such that $F$ is identically 0 on $\{(n, m) \in B \times B: n<m\}$, or else $n_{i} \in A$ for $i<\alpha$ such that $n_{j}<n_{i}$ for all $j<i$, and $F$ is identically 1 on $\left\{\left(n_{j}, n_{i}\right): j<i<\alpha\right\}$.

- $K^{+} \nrightarrow\left(K^{+}, \alpha\right)^{2}$ is the negation of $K^{+} \rightarrow\left(K^{+}, \alpha\right)^{2}$.

- For any set $A, P_{\omega}(A)$ denotes the collection of all finite subsets of $A$.

- $\lambda$ is a fixed infinite cardinal.

- $\widehat{a}=\left\{b \in P_{\omega}(\lambda): a \subseteq b\right\}$ for every $a \in P_{\omega}(\lambda)$.

- $I_{\omega, \lambda}$ denotes the set of all $A \subseteq P_{\omega}(\lambda)$ such that $A \cap \widehat{a}=\emptyset$ for some $a \in P_{\omega}(\lambda)$.

- An ideal $J$ on $P_{\omega}(\lambda)$ is fine if $I_{\omega, \lambda} \subseteq J$. It is easy to see that $\operatorname{cof}(J) \geq \lambda$ for every fine ideal $J$ on $P_{\omega}(\lambda)$. As is readily verified, $I_{\omega, \lambda}$ is a fine ideal on $P_{\omega}(\lambda)$ and $\operatorname{cof}\left(I_{\omega, \lambda}\right)=\lambda$. 
- Given two sets $X$ and $Y, Y^{X}$ denotes the set of all functions from $X$ to $Y$. We endow the set $2^{\omega}$ with the product topology, where 2 is given the discrete topology.

- $O_{s}=\left\{f \in 2^{\omega}: s \subset f\right\}$ for all $s \in \bigcup_{n \in \omega} 2^{n}$.

- $M$ denotes the collection of all meager subsets of $2^{\omega}$.

- $\operatorname{cov}(M)$ is the least cardinality of any $X \subseteq M$ such that $2^{\omega}=\cup X$.

- $\mathfrak{d}$ is the least cardinality of any $F \subseteq \omega^{\omega}$ with the property that for every $g \in \omega^{\omega}$, there is $f \in F$ such that $g(n) \leq f(n)$ for all $n \in \omega$. It is well known (see e.g. [15]) that $\omega_{1} \leq \operatorname{cov}(M) \leq \mathfrak{d}$.

- $\mathfrak{d}_{\omega, \lambda}^{\omega}$ is the least cardinality of any family $F$ of functions from $\omega$ to $P_{\omega}(\lambda)$ with the property that for every $g: \omega \rightarrow P_{\omega}(\lambda)$, there is $f \in F$ such that $g(n) \subseteq f(n)$ for all $n \in \omega$. It is shown in [12] that $\mathfrak{d}_{\omega, \omega}^{\omega}=\mathfrak{d}$, and that $\mathfrak{d}_{\omega, \lambda}^{\omega}=\max \left\{\mathfrak{d}, u\left(\omega_{1}, \lambda\right)\right\}$ if $\lambda>\omega$, where $u\left(\omega_{1}, \lambda\right)$ is the least cardinality of any family $X$ of countable subsets of $\lambda$ such that for every countable $a \subseteq \lambda$, there is $b \in X$ with $a \subseteq b$.

2. Weak $\chi$-points. In this section we introduce the property of being a weak $\chi$-point and determine when $I_{\omega, \lambda}$ has this property.

An ideal $J$ on $P_{\omega}(\lambda)$ is a weak $\chi$-point if given $A \in J^{+}$and $g: \omega \rightarrow P_{\omega}(\lambda)$, there is $C \in J^{+} \cap P(A)$ such that $g(\max (a \cap \omega)) \subseteq b$ for all $a, b \in C$ with $\max (a \cap \omega)<\max (b \cap \omega)$.

Lemma 2.1. Let $J$ be a fine ideal on $P_{\omega}(\lambda)$ such that $\operatorname{cof}(J)<\operatorname{cov}(M)$. Then $J$ is a weak $\chi$-point.

Proof. Fix $A \in J^{+}$and $g: \omega \rightarrow P_{\omega}(\lambda)$. Set

$$
A_{n}=\{a \in A: \max (a \cap \omega)=n\}
$$

for each $n \in \omega$. Pick $B_{\alpha} \in J$ for $\alpha<\operatorname{cof}(J)$ so that $J=\bigcup_{\alpha<\operatorname{cof}(J)} P\left(B_{\alpha}\right)$. For $\alpha<\operatorname{cof}(J)$ and $n \in \omega$, let $D_{\alpha}^{n}$ be the set of all $s \in 2^{n+1}$ such that $s(n)=1$ and there is $a \in A_{n}-B_{\alpha}$ with the property that $g(m) \subseteq a$ for all $m<n$ with $s(m)=1$. Given $\alpha<\operatorname{cof}(J)$, let $D_{\alpha}=\bigcup_{n \in \omega} D_{\alpha}^{n}$ and $U_{\alpha}=\bigcup_{s \in D_{\alpha}} O_{s}$. Let us show that the open set $U_{\alpha}$ is dense. Thus let $k \in \omega$ and $p \in 2^{k}$. Put $y=\{m<k: p(m)=1\}$. Pick $b \in A-B_{\alpha}$ so that $\bigcup_{m \in y} g(m) \subseteq b$ and $k \leq \max (b \cap \omega)$. Now define $q \supset p$ by $\operatorname{dom}(q)=\max (b \cap \omega)+1$, $q(\max (b \cap \omega))=1$ and $q(i)=0$ for all $i$ with $k \leq i<\max (b \cap \omega)$. Then clearly $q \in D_{\alpha}^{\max (b \cap \omega)}$.

Now select $f \in \bigcap_{\alpha<\operatorname{cof}(J)} U_{\alpha}$. For each $\alpha<\operatorname{cof}(J)$, pick $s_{\alpha} \in D_{\alpha}$ with $s_{\alpha} \subset f$. Put

$$
Y=\left\{\max \left(\operatorname{dom}\left(s_{\alpha}\right)\right): \alpha<\operatorname{cof}(J)\right\}
$$

and let $m_{0}, m_{1}, \ldots$ be the increasing enumeration of $Y$. Set $E_{0}=A_{m_{0}}$ and 
for each $l \in \omega$,

$$
E_{l+1}=\left\{a \in A_{m_{l+1}}: \bigcup_{i \leq l} g\left(m_{i}\right) \subseteq a\right\} .
$$

Finally define $C=\bigcup_{l \in \omega} E_{l}$. Given $\alpha<\operatorname{cof}(J)$, let $l$ be such that $\max \left(\operatorname{dom}\left(s_{\alpha}\right)\right)=m_{l}$. Then $E_{l}-B_{\alpha} \neq \emptyset$ since $s_{\alpha} \in D_{\alpha}^{m_{l}}$ and $s_{\alpha}\left(m_{i}\right)=1$ for all $i<l$. Thus $C \in J^{+}$.

We will need the following result from [10].

LEMMA 2.2. $\operatorname{cov}(M)$ is the least cardinal $\mu$ with the property that there is an ideal $K$ on $\omega$ such that $\operatorname{cof}(K)=\mu$ and $K$ is not weakly selective.

Proposition 2.3. $I_{\omega, \lambda}$ is a weak $\chi$-point if and only if $\lambda<\operatorname{cov}(M)$.

Proof. The right-to-left direction is immediate from Lemma 2.1. For the other implication, assume $\lambda>\omega$ and $I_{\omega, \lambda}$ is a weak $\chi$-point. We will show that every ideal $K$ on $\omega$ such that $\operatorname{cof}(K) \leq \lambda$ is weakly selective. This will give $\lambda<\operatorname{cov}(M)$ by Lemma 2.2 .

Thus let $K$ be a fixed ideal on $\omega$ with $\operatorname{cof}(K) \leq \lambda$, and let $A \in K^{+}$. Pick $x \subseteq \lambda-\omega$ with $|x|=\operatorname{cof}(K)$, and a one-to-one $h: x \rightarrow K$ with the property that $K=\bigcup_{\beta \in x} P(h(\beta))$. For each $n \in A$, let $X_{n}$ be the set of all $a \in P_{\omega}(\lambda)$ such that $a \cap \omega=n+1$ and $a \cap x \subseteq\{\alpha \in x: n \notin h(\alpha)\}$. We let $B=\bigcup_{n \in A} X_{n}$. Given $d \in P_{\omega}(\lambda)$, we have $\bigcup_{\beta \in d \cap x} h(\beta) \in K$ and therefore there is $k \in A$ such that $k \geq \max (d \cap \omega)$ and $k \notin \bigcup_{\beta \in d \cap x} h(\beta)$. Setting $c=(k+1) \cup(d-\omega)$, we have $d \subseteq c$ and $c \in X_{k}$. Hence $B \in I_{\omega, \lambda}^{+}$.

Now let $E_{n} \in K$ for $n \in \omega$. Define $p: \omega \rightarrow x$ so that $E_{n} \subseteq h(p(n))$ for all $n \in \omega$. Since $I_{\omega, \lambda}$ is a weak $\chi$-point, there is $C \in I_{\omega, \lambda}^{+} \cap P(B)$ such that $p(\max (b \cap \omega)) \in e$ for all $b, e \in C$ with $\max (b \cap \omega)<\max (e \cap \omega)$. Set $D=\{\max (a \cap \omega): a \in C\}$. Given $\alpha \in x$, there is $a \in C$ such that $\alpha \in a$. Then $\max (a \cap \omega) \notin h(\alpha)$. Hence $D \in K^{+}$. Moreover, $D \subseteq A$. Finally let $b, e \in C$ with $\max (b \cap \omega)<\max (e \cap \omega)$. As $p(\max (b \cap \omega)) \in e$, we have $\max (e \cap \omega) \notin h(p(\max (b \cap \omega)))$ and therefore $\max (e \cap \omega) \notin E_{\max (b \cap \omega)}$.

3. $J^{+} \stackrel{\omega}{\longrightarrow}\left(J^{+}\right)^{2}$. We now introduce the partition property $J^{+} \stackrel{\omega}{\longrightarrow}\left(J^{+}\right)^{2}$ and show that it is satisfied whenever $J$ has a small (meaning $<\operatorname{cov}(M)$ ) number of generators. We start with a few definitions.

Let $J$ be an ideal on $P_{\omega}(\lambda)$.

- $J^{+} \stackrel{\omega}{\longrightarrow}\left(J^{+}\right)^{2}$ asserts that given $A \in J^{+}$and $F: \omega \times P_{\omega}(\lambda) \rightarrow 2$, there is $B \in J^{+} \cap P(A)$ such that $F$ is constant on

$$
\{(\max (a \cap \omega), b) \in \omega \times B: a \in B \text { and } \max (a \cap \omega)<\max (b \cap \omega)\} .
$$


- $J$ is almost $(\omega, 2)$-distributive if given $A \in J^{+}$and $B_{n} \subseteq P_{\omega}(\lambda)$ for $n \in \omega$, there is $C \in J^{+} \cap P(A)$ such that

for all $a \in C$.

$$
\left\{C-B_{\max (a \cap \omega)}, C \cap B_{\max (a \cap \omega)}\right\} \cap J \neq \emptyset
$$

- $J$ is a weak $\pi$-point if given $A \in J^{+}$and $B_{n} \in J$ for $n \in \omega$, there is $C \in J^{+} \cap P(A)$ such that $C \cap B_{n} \in I_{\omega, \lambda}$ for all $n \in \omega$.

Lemma 3.1. Let $J$ be a fine ideal on $P_{\omega}(\lambda)$. Then the following are equivalent:

(i) $J^{+} \stackrel{\omega}{\rightarrow}\left(J^{+}\right)^{2}$.

(ii) $J$ is almost $(\omega, 2)$-distributive and both a weak $\chi$-point and a weak $\pi$-point.

Proof. (i) $\Rightarrow$ (ii). Assume $J^{+} \stackrel{\omega}{\rightarrow}\left(J^{+}\right)^{2}$. Then given $A \in J^{+}$and $B_{n} \subseteq$ $P_{\omega}(\lambda)$ for $n \in \omega$, there is $C \in J^{+} \cap P(A)$ such that either $b \in B_{\max (a \cap \omega)}$ for all $a, b \in C$ with $\max (a \cap \omega)<\max (b \cap \omega)$, or else $b \notin B_{\max (a \cap \omega)}$ for all $a, b \in C$ with $\max (a \cap \omega)<\max (b \cap \omega)$. It easily follows that (ii) holds.

(ii) $\Rightarrow$ (i). Assume (ii), and fix $A \in J^{+}$and $F: \omega \times P_{\omega}(\lambda) \rightarrow 2$. For $n \in \omega$ and $i<2$, set $B_{n}^{i}=\{b \in A: F(n, b)=i\}$. Since $J$ is almost $(\omega, 2)$ distributive, there are $C \in J^{+} \cap P(A)$ and $h:\{\max (a \cap \omega): a \in C\} \rightarrow 2$ with $C-B_{\max (a \cap \omega)}^{h(\max (a \cap \omega))} \in J$ for all $a \in C$. Set $C_{i}=\{a \in C: h(\max (a \cap \omega))=i\}$ for each $i<2$. Pick $j<2$ so that $C_{j} \in J^{+}$. Since $J$ is a weak $\pi$-point and a weak $\chi$-point, there is $D \in J^{+} \cap P\left(C_{j}\right)$ such that $b \in B_{\max (a \cap \omega)}^{j}$ for all $a, b \in D$ with $\max (a \cap \omega)<\max (b \cap \omega)$. Then $F$ takes the constant value $j$ on

$$
\{(\max (a \cap \omega), b) \in \omega \times D: a \in D \text { and } \max (a \cap \omega)<\max (b \cap \omega)\} .
$$

The following is proved in [11].

Lemma 3.2. Let $J$ be a fine ideal on $P_{\omega}(\lambda)$ such that $\operatorname{cof}(J)<\mathfrak{d}_{\omega, \lambda}^{\omega}$. Then $J$ is almost $(\omega, 2)$-distributive and a weak $\pi$-point.

Proposition 3.3. Let $J$ be a fine ideal on $P_{\omega}(\lambda)$ such that $\operatorname{cof}(J)<$ $\operatorname{cov}(M)$. Then $J^{+} \stackrel{\omega}{\rightarrow}\left(J^{+}\right)^{2}$.

Proof. By Lemmas 2.1, 3.1 and 3.2.

The following is immediate from Proposition 3.3.

Corollary 3.4. If $\lambda<\operatorname{cov}(M)$, then $I_{\omega, \lambda}^{+} \stackrel{\omega}{\rightarrow}\left(I_{\omega, \lambda}^{+}\right)^{2}$.

4. $I_{\omega, \lambda}^{+} \underset{\omega}{\stackrel{\omega}{\rightarrow}}\left(I_{\omega, \lambda}^{+}, \alpha\right)^{2}$. This section deals with negative partition properties. Let $J$ be an ideal on $P_{\omega}(\lambda)$ and $\alpha$ an ordinal with $2 \leq \alpha \leq \omega$. 
- $J^{+} \underset{\omega}{\stackrel{\omega}{\omega}}\left(J^{+}, \alpha\right)^{2}$ means that for all $A \in J^{+}$and $f: \omega \times \omega \rightarrow 2$, either there is $B \in J^{+} \cap P(A)$ such that $f$ is identically 0 on

$$
\{(\max (a \cap \omega), \max (b \cap \omega)): a, b \in B \text { and } \max (a \cap \omega)<\max (b \cap \omega)\},
$$

or there are $a_{n} \in A$ for $n<\alpha$ such that $\max \left(a_{m} \cap \omega\right)<\max \left(a_{n} \cap \omega\right)$ for all $m<n$, and $f$ is identically 1 on $\left\{\left(\max \left(a_{m} \cap \omega\right), \max \left(a_{n} \cap \omega\right)\right): m<n<\alpha\right\}$.

- $J^{+} \underset{\omega}{\stackrel{\omega}{\omega}}\left(J^{+}, \alpha\right)^{2}$ means that $J^{+} \underset{\omega}{\omega}\left(J^{+}, \alpha\right)^{2}$ does not hold.

Lemma 4.1. Let $\alpha$ be such that $2 \leq \alpha \leq \omega$ and $I_{\omega, \lambda}^{+} \underset{\omega}{\stackrel{\omega}{\omega}}\left(I_{\omega, \lambda}^{+}, \alpha\right)^{2}$, and let $K$ be an ideal on $\omega$ with $\operatorname{cof}(K) \leq \lambda$. Then $K^{+} \rightarrow\left(K^{+}, \alpha\right)^{2}$.

Proof. Fix $A \in K^{+}$and $f: \omega \times \omega \rightarrow 2$. Let $B$ be defined as in the proof of Proposition 2.3. If $f$ is identically 0 on

$\{(\max (a \cap \omega), \max (b \cap \omega)): a, b \in C$ and $\max (a \cap \omega)<\max (b \cap \omega)\}$

for some $C \in I_{\omega, \lambda}^{+} \cap P(B)$, then setting $D=\{\max (a \cap \omega): a \in C\}$, we see that $D \in K^{+} \cap P(A)$ and $f$ is identically 0 on $\{(n, m) \in D \times D: n<m\}$. On the other hand, if $f$ is identically 1 on $\left\{\left(\max \left(a_{p} \cap \omega\right), \max \left(a_{q} \cap \omega\right)\right): p<q<\alpha\right\}$, where $\left\{a_{q}: q<\alpha\right\} \subseteq B$ and $\max \left(a_{p} \cap \omega\right)<\max \left(a_{q} \cap \omega\right)$ whenever $p<q<\alpha$, then setting $E=\left\{\max \left(a_{q} \cap \omega\right): q<\alpha\right\}$, we find that $E \subseteq A,|E|=\alpha$ and $f$ is identically 1 on $\{(n, m) \in E \times E: n<m\}$.

The following result is folklore. As we do not know any explicit reference for it, a proof is provided.

Lemma 4.2. Given an ideal $K$ on $\omega$, the following are equivalent:

(i) $K$ is weakly selective.

(ii) $K^{+} \rightarrow\left(K^{+}, \omega\right)^{2}$.

Proof. (i) $\Rightarrow$ (ii). Assume (i) and fix $A \in K^{+}$and $F: \omega \times \omega \rightarrow 2$. Set $E_{n}=$ $\{m>n: F(n, m)=1\}$ for all $n \in \omega$. First suppose there is $B \in K^{+} \cap P(A)$ such that $B \cap E_{n} \in K$ for every $n \in B$. Pick $C \in K^{+} \cap P(B)$ so that $m \notin B \cap E_{n}$ for all $n, m \in C$ with $n<m$. Then $F$ takes the constant value 0 on $\{(n, m) \in C \times C: n<m\}$. Now suppose there is $\varphi: K^{+} \cap P(A) \rightarrow A$ such that $\varphi(D) \in D$ and $D \cap E_{\varphi(D)} \in K^{+}$for all $D \in K^{+} \cap P(A)$. Define $n_{i} \in A$ for $i<\omega$ by $n_{0}=\varphi(A)$ and $n_{i+1}=\varphi\left(A \cap \bigcap_{j \leq i} E_{n_{j}}\right)$. Then $F$ is identically 1 on $\left\{\left(n_{j}, n_{i}\right): j<i<\omega\right\}$.

(ii) $\Rightarrow$ (i). Assume (ii) and fix $A \in K^{+}$and $B_{i} \in K$ for $i<\omega$. We must find $H \in K^{+} \cap P(A)$ such that $m \notin B_{n}$ for all $n, m \in H$ with $n<m$. If $A-\bigcup_{i<\omega} B_{i} \in K^{+}$, we can set $H=A-\bigcup_{i<\omega} B_{i}$. Otherwise we put $C=A \cap \bigcup_{i<\omega} B_{i}$ and define $h: C \rightarrow \omega$ by $h(n)=$ the least $i$ such that $n \in B_{i}$. Now define $F: C \times C \rightarrow 2$ by $F(n, m)=1$ precisely when $h(n)>h(m)$. Then clearly, there is $D \in K^{+} \cap P(C)$ such that $F$ is identically 0 on 
$\{(n, m) \in D \times D: n<m\}$. Notice that $D \cap B_{i}$ is finite for each $i<\omega$, since $D \cap B_{i} \subseteq m$ whenever $m \in D-\bigcup_{j \leq i} B_{j}$. Finally, define $G: D \times D \rightarrow 2$ by $G(n, m)=1$ if and only if $m \in B_{n}$. Clearly, there is $H \in K^{+} \cap P(D)$ such that $F$ takes the constant value 0 on $\{(n, m) \in H \times H: n<m\}$. Then $H$ is as desired.

The following shows that Proposition 3.3 is optimal.

Proposition 4.3. If $\lambda \geq \operatorname{cov}(M)$, then $I_{\omega, \lambda}^{+} \underset{\omega}{\stackrel{\omega}{\omega}}\left(I_{\omega, \lambda}^{+}, \omega\right)^{2}$.

Proof. By Lemmas 2.2, 4.1 and 4.2.

For each ordinal $\alpha$ with $3 \leq \alpha \leq \omega$, let $\mathfrak{p a r}_{\alpha}$ be the least cardinal $\mu$ with the property that there is an ideal $K$ on $\omega$ such that $\operatorname{cof}(K)=\mu$ and $K^{+} \nrightarrow\left(K^{+}, \alpha\right)^{2}$.

It follows from Lemmas 2.2 and 4.2 that $\mathfrak{p a r}_{\omega}=\operatorname{cov}(M)$. The exact value of $\mathfrak{p a r}_{3}$ is not known, but one has the following upper bound (see [4], p. 63, and [2], p. 7).

Proposition 4.4. $\mathfrak{p a r}_{3} \leq \mathfrak{d}$.

Proof. Fix a bijection $j: \omega \times \omega \times \omega \rightarrow \omega$, and let $K$ be the set of all $B \subseteq \omega$ such that

$\{m \in \omega:\{n \in \omega:\{p \in \omega: j(m, n, p) \in B\}$ is infinite $\}$ is infinite $\}$ is finite.

It is easy to check that $K$ is an ideal on $\omega$. To see that $K^{+} \nrightarrow\left(K^{+}, 3\right)^{2}$, consider $F: \omega \times \omega \rightarrow 2$ defined by $F\left(j(m, n, p), j\left(m^{\prime}, n^{\prime}, p^{\prime}\right)\right)=1$ if and only if $m<n<m^{\prime}<p<n^{\prime}<p^{\prime}$.

It remains to check that $\operatorname{cof}(K) \leq \mathfrak{d}$. Select $X \subseteq \omega^{\omega}$ so that for every $f \in \omega^{\omega}$, there is $g \in X$ with the property that $f(n) \leq g(n)$ for all $n \in \omega$. Fix a bijection $k: \omega \times \omega \rightarrow \omega$. For $m \in \omega$ and $f, g \in X$, set

$$
\begin{aligned}
A_{m} & =j[m \times \omega \times \omega], \\
B_{m, f} & =\bigcup_{n \geq m} j[\{n\} \times f(n) \times \omega], \\
C_{m, f, g} & =\bigcup_{n \geq m} \bigcup_{p \geq f(n)} j[\{n\} \times\{p\} \times g(k(n, p))], \\
D_{m, f, g} & =A_{m} \cup B_{m, f} \cup C_{m, f, g} .
\end{aligned}
$$

It is readily verified that $K=\bigcup\left\{P\left(D_{m, f, g}\right): m \in \omega\right.$ and $\left.f, g \in X\right\}$.

Jörg Brendle has shown that the inequality in Proposition 4.4 can consistently be strict. His result is included here with his kind permission.

Proposition 4.5. It is consistent with ZFC that $\mathfrak{p a r}_{3}<\mathfrak{d}$.

Proof. Let $V \vDash \mathrm{ZFC}+\mathrm{GCH}$. By a result of Baumgartner and Taylor (Corollary 4.12 in [3]), there is in $V$ a prime $P$-point ideal $K$ on $\omega$ such 
that $K^{+} \nrightarrow\left(K^{+}, 3\right)^{2}$. Now let $Q$ be an $\omega_{2}$-stage countable-support iteration of Miller's rational perfect set forcing. In $V^{Q}$ the following hold (see [5]): (a) $\mathfrak{d}=\aleph_{2}=2^{\aleph_{0}}$, and (b) $J=\bigcup_{B \in K} P(B)$ is a prime ideal on $\omega$. Clearly, $\operatorname{cof}(J)=\aleph_{1}$. Moreover, $J^{+} \nrightarrow\left(J^{+}, 3\right)^{2}$.

Proposition 4.6. If $\lambda \geq \mathfrak{p a r}_{3}$, then $I_{\omega, \lambda}^{+} \underset{\omega}{\stackrel{\omega}{\leftrightarrow}}\left(I_{\omega, \lambda}^{+}, 3\right)^{2}$.

Proof. By Lemma 4.1.

5. Extending ideals. Suppose we are given a property $P$ of ideals and a cardinal $\mu>\lambda$. Then one might ask whether it is possible to extend every fine ideal $J$ on $P_{\omega}(\lambda)$ such that $\operatorname{cof}(J)<\mu$ to an ideal $K$ on $P_{\omega}(\lambda)$ with the property $P$. In this section we will consider several questions of this type. We start with a lemma.

Lemma 5.1. There is a fine ideal $J$ on $P_{\omega}(\lambda)$ such that $\operatorname{cof}(J)=$ $\max \{\lambda, \operatorname{cov}(M)\}$ and $J^{+} \underset{\omega}{\stackrel{\omega}{\omega}}\left(J^{+}, \omega\right)^{2}$.

Proof. By Lemmas 2.2 and 4.2, we can find an ideal $K$ on $\omega$ with $\operatorname{cof}(K)=\operatorname{cov}(M), E \in K^{+}$and $f: \omega \times \omega \rightarrow 2$ such that (a) there is no $B \in K^{+} \cap P(E)$ such that $f$ is identically 0 on $\{(n, m) \in B \times B: n<m\}$, and (b) there is no infinite subset $C$ of $E$ such that $f$ is identically 1 on $\{(n, m) \in C \times C: n<m\}$. Define $\varphi: E \times P\left(P_{\omega}(\lambda)\right) \rightarrow P\left(P_{\omega}(\lambda)\right)$ by letting $\varphi(n, A)=\{b \in A: \max (b \cap \omega)=n\}$. For $a \in P_{\omega}(\lambda)$ and $A \subseteq P_{\omega}(\lambda)$, set $Y_{A}^{a}=\{n \in E: \widehat{a} \cap \varphi(n, A) \neq \emptyset\}$. Now define $J \subseteq P\left(P_{\omega}(\lambda)\right)$ by letting $A \in J$ if and only if $Y_{A}^{a} \in K$ for some $a \in P_{\omega}(\lambda)$. It is immediate from the following easy facts that $J$ is a fine ideal on $P_{\omega}(\lambda)$ :

(i) the set $E-Y_{P_{\omega}(\lambda)}^{a}$ is finite for all $a \in P_{\omega}(\lambda)$;

(ii) $Y_{A}^{a} \subseteq Y_{B}^{a}$ for all $a \in P_{\omega}(\lambda)$ and $A, B \subseteq P_{\omega}(\lambda)$ with $A \subseteq B$;

(iii) $Y_{A \cup B}^{a \cup b} \subseteq Y_{A}^{a} \cup Y_{B}^{b}$ for all $a, b \in P_{\omega}(\lambda)$ and $A, B \subseteq P_{\omega}(\lambda)$;

(iv) if $A \in I_{\omega, \lambda}$, then $Y_{A}^{a}=\emptyset$ for some $a \in P_{\omega}(\lambda)$.

Set $A=\left\{a \in P_{\omega}(\lambda): \max (a \cap \omega) \in E\right\}$. Then $A \in J^{*}$, as $Y_{P_{\omega}(\lambda)-A}^{\emptyset}=\emptyset$. Given $B \in J^{+} \cap P(A)$, set $C=\{\max (a \cap \omega): a \in B\}$. Then $C \in K^{+}$since $C=Y_{B}^{\emptyset}$, and therefore $f$ is not constantly 0 on

$$
\{(\max (a \cap \omega), \max (b \cap \omega)): a, b \in B \text { and } \max (a \cap \omega)<\max (b \cap \omega)\} .
$$

It easily follows that $J^{+} \underset{\omega}{\stackrel{\omega}{\omega}}\left(J^{+}, \omega\right)^{2}$.

It remains to compute $\operatorname{cof}(J)$. Given $D \subseteq P_{\omega}(\lambda)$, we know that $D \in J$ if and only if there are $a \in P_{\omega}(\lambda)$ and $H \in K$ such that

$$
D \subseteq\left(P_{\omega}(\lambda)-\widehat{a}\right) \cup\left\{b \in P_{\omega}(\lambda): \max (b \cap \omega) \in H \cup(\omega-E)\right\} .
$$


It clearly follows that $\operatorname{cof}(J) \leq \max \{\lambda, \operatorname{cof}(K)\}$. On the other hand, $\operatorname{cof}(J) \geq \operatorname{cov}(M)$ by Proposition 3.3. Hence $\operatorname{cof}(J)=\max \{\lambda, \operatorname{cov}(M)\}$.

Ketonen [7] showed that if $\operatorname{cov}(M)=2^{\aleph_{0}}$, then every ideal on $\omega$ generated by less than $2^{\aleph_{0}}$ sets can be extended to a prime ideal $K$ on $\omega$ such that $K^{+} \rightarrow\left(K^{+}\right)^{2}$. The converse was proved by Canjar [6] and by Bartoszyński and Judah [1]. The equivalence can be generalized as follows.

Proposition 5.2. The following are equivalent:

(i) $\operatorname{cov}(M)=2^{\lambda}$.

(ii) If $J$ is a fine ideal on $P_{\omega}(\lambda)$ with $\operatorname{cof}(J)<2^{\lambda}$, then there is a prime ideal $K$ on $P_{\omega}(\lambda)$ such that $J \subseteq K$ and $K^{+} \stackrel{\omega}{\rightarrow}\left(K^{+}\right)^{2}$.

(iii) If $J$ is a fine ideal on $P_{\omega}(\lambda)$ with $\operatorname{cof}(J)<2^{\lambda}$, then there is an ideal $K$ on $P_{\omega}(\lambda)$ such that $J \subseteq K$ and $K^{+} \underset{\omega}{\stackrel{\omega}{\longrightarrow}}\left(K^{+}, \omega\right)^{2}$.

Proof. (i) $\Rightarrow$ (ii). Assume $\operatorname{cov}(M)=2^{\lambda}$, and let $J$ be a fine ideal on $P_{\omega}(\lambda)$ with $\operatorname{cof}(J)<2^{\lambda}$. Let $F_{\alpha}$ for $\alpha<2^{\lambda}$ be an enumeration of the set of all $F: \omega \times P_{\omega}(\lambda) \rightarrow 2$. Using Proposition 3.3, we define for each $\alpha<2^{\lambda}$ a fine ideal $J_{\alpha}$ on $P_{\omega}(\lambda)$ with $\operatorname{cof}\left(J_{\alpha}\right) \leq \max \{|\alpha|, \operatorname{cof}(J)\}$, and $A_{\alpha} \in J_{\alpha}^{+}$so that

(0) $J_{0}=J$

(1) $F_{\alpha}$ is constant on

$\left\{(\max (a \cap \omega), b) \in \omega \times A_{\alpha}: a \in A_{\alpha}\right.$ and $\left.\max (a \cap \omega)<\max (b \cap \omega)\right\} ;$

(2) $J_{\alpha+1}=J_{\alpha} \mid A_{\alpha}$;

(3) $J_{\alpha}=\bigcup_{\beta<\alpha} J_{\beta}$ if $\alpha$ is a limit ordinal $>0$.

Finally set $K=\bigcup_{\alpha<2^{\lambda}} J_{\alpha}$. Then clearly $K$ is an ideal on $P_{\omega}(\lambda)$ such that $J \subseteq K$. Moreover, as $A_{\alpha} \in K^{*}$ for every $\alpha<2^{\lambda}$, we conclude that $K^{+} \stackrel{\omega}{\rightarrow}\left(K^{+}\right)^{2}$ and $K$ is prime.

(ii) $\Rightarrow$ (iii). Trivial.

(iii) $\Rightarrow\left(\right.$ i). Assume $\operatorname{cov}(M) \neq 2^{\lambda}$. Then $\operatorname{cov}(M)<2^{\lambda}$ because $\operatorname{cov}(M)$ $\leq 2^{\aleph_{0}}$. By Lemma 5.1 one can find a fine ideal $J$ on $P_{\omega}(\lambda)$ with $\operatorname{cof}(J)=$ $\max \{\lambda, \operatorname{cov}(M)\}, A \in J^{+}$and $F: \omega \times \omega \rightarrow 2$ with the property that (a) there is no $C \in J^{+} \cap P(A)$ such that $F$ is constantly 0 on

$$
\{(\max (a \cap \omega), \max (b \cap \omega)): a, b \in C \text { and } \max (a \cap \omega)<\max (b \cap \omega)\},
$$

and (b) there is no $f \in A^{\omega}$ such that $\max (f(n) \cap \omega)<\max (f(m) \cap \omega)$ whenever $n<m<\omega$, and $F$ is identically 1 on

$$
\{(\max (f(n) \cap \omega), \max (f(m) \cap \omega)): n<m<\omega\} .
$$

We have $\operatorname{cof}(J \mid A)<2^{\lambda}$. Moreover, $K^{+} \underset{\omega}{\stackrel{\omega}{\omega}}\left(K^{+}, \omega\right)^{2}$ for every ideal $K$ on $P_{\omega}(\lambda)$ with $J \mid A \subseteq K$. 
Canjar [6] showed that $\operatorname{cov}(M)=\mathfrak{d}$ if and only if every ideal on $\omega$ generated by less than $\mathfrak{d}$ sets can be extended to a $Q$-point. We will now generalize his result.

An ideal $J$ on $P_{\omega}(\lambda)$ is a $\chi$-point if for every $g: \omega \rightarrow P_{\omega}(\lambda)$, there is $A \in J^{*}$ such that $g(\max (a \cap \omega)) \subseteq b$ for all $a, b \in A$ with $\max (a \cap \omega)<$ $\max (b \cap \omega)$.

Notice that if an ideal $J$ on $P_{\omega}(\lambda)$ is a $\chi$-point, then so is every ideal $K$ on $P_{\omega}(\lambda)$ with $J \subseteq K$.

Proposition 5.3. Let $J$ be a fine ideal on $P_{\omega}(\lambda)$ that is a $\chi$-point. Then $\operatorname{cof}(J) \geq \mathfrak{d}_{\omega, \lambda}^{\omega}$.

Proof. Let $X \subseteq J^{*}$ be such that $J=\bigcup_{A \in X} P\left(P_{\omega}(\lambda)-A\right)$. Given $A \in X$, we define $f_{A}: \omega \rightarrow P_{\omega}(\lambda)$ as follows. Let $m_{0}^{A}, m_{1}^{A}, \ldots$ be the increasing enumeration of the elements of the set $\{\max (a \cap \omega): a \in A\}$. For each $n \in \omega$, pick $a_{n}^{A} \in A$ with $\max \left(a_{n}^{A} \cap \omega\right)=m_{n}^{A}$. We put $f_{A}(n)=a_{n+1}^{A}$ for all $n \in \omega$.

Now fix $g: \omega \rightarrow P_{\omega}(\lambda)$. Define $h: \omega \rightarrow P_{\omega}(\lambda)$ by $h(m)=\bigcup_{n \leq m} g(n)$. Select $A \in X$ so that $h(\max (a \cap \omega)) \subseteq b$ for all $a, b \in A$ satisfying $\max (a \cap \omega)<\max (b \cap \omega)$. For each $n \in \omega$, we have $g(n) \subseteq h\left(m_{n}^{A}\right) \subseteq f_{A}(n)$. Thus $|X| \geq \mathfrak{d}_{\omega, \lambda}^{\omega}$.

The proof of the following shows that assuming $\lambda<\operatorname{cov}(M)=\mathfrak{d}_{\omega, \lambda}^{\omega}$, there is a fine ideal $J$ on $P_{\omega}(\lambda)$ such that $J$ is a $\chi$-point and $\operatorname{cof}(J)=\mathfrak{d}_{\omega, \lambda}^{\omega}$.

Proposition 5.4. Assuming $\lambda<\mathfrak{d}_{\omega, \lambda}^{\omega}$ the following are equivalent:

(i) $\operatorname{cov}(M)=\mathfrak{d}_{\omega, \lambda}^{\omega}$.

(ii) If $J$ is a fine ideal on $P_{\omega}(\lambda)$ with $\operatorname{cof}(J)<\mathfrak{d}_{\omega, \lambda}^{\omega}$, then there is a $\chi$-point ideal $K$ on $P_{\omega}(\lambda)$ such that $J \subseteq K$.

Proof. (i) $\Rightarrow$ (ii). Assume (i), and let $J$ be a fine ideal on $P_{\omega}(\lambda)$ with $\operatorname{cof}(J)<\mathfrak{d}_{\omega, \lambda}^{\omega}$. Pick $f_{\alpha}: \omega \rightarrow P_{\omega}(\lambda)$ for $\alpha<\operatorname{cov}(M)$ so that for every $g: \omega \rightarrow P_{\omega}(\lambda)$, there is $\alpha<\operatorname{cov}(M)$ such that $g(n) \subseteq f(n)$ for all $n \in \omega$. Using Lemma 2.1, define for each $\alpha<\operatorname{cov}(M)$ an ideal $J_{\alpha}$ on $P_{\omega}(\lambda)$ with $\operatorname{cof}\left(J_{\alpha}\right) \leq \max \{|\alpha|, \operatorname{cof}(J)\}$, and $A_{\alpha} \in J_{\alpha}^{+}$so that

(0) $J_{0}=J$

(1) $f_{\alpha}(\max (a \cap \omega)) \subseteq b$ for all $a, b \in A_{\alpha}$ with $\max (a \cap \omega)<\max (b \cap \omega)$;

(2) $J_{\alpha+1}=J_{\alpha} \mid A_{\alpha}$;

(3) $J_{\alpha}=\bigcup_{\beta<\alpha} J_{\beta}$ if $\alpha$ is a limit ordinal $>0$.

Setting $K=\bigcup_{\alpha<\operatorname{cov}(M)} J_{\alpha}$, we clearly find that $K$ is a $\chi$-point ideal on $P_{\omega}(\lambda)$ extending $J$.

(ii) $\Rightarrow$ (i). Assume (i) does not hold. Then $\operatorname{cov}(M)<\mathfrak{d}_{\omega, \lambda}^{\omega}$ since $\operatorname{cov}(M) \leq$ $\mathfrak{d} \leq \mathfrak{d}_{\omega, \lambda}^{\omega}$. Hence by Lemma 5.1 , there is a fine ideal $J$ on $P_{\omega}(\lambda)$ such that 
$\operatorname{cof}(J)<\mathfrak{d}_{\omega, \lambda}^{\omega}$ and $J^{+} \stackrel{\omega}{\leftrightarrow}\left(J^{+}\right)^{2} . J$ is not a weak $\chi$-point by Lemmas 3.1 and 3.2, and so we can find $A \in J^{+}$and $g: \omega \rightarrow P_{\omega}(\lambda)$ with the property that there is no $B \in J^{+} \cap P(A)$ such that $g(\max (a \cap \omega)) \subseteq b$ for all $a, b \in B$ with $\max (a \cap \omega)<\max (b \cap \omega)$. Then there is no weak $\chi^{-}$ point ideal $K$ on $P_{\omega}(\lambda)$ extending $J \mid A$. We have $\operatorname{cof}(J \mid A)<\mathfrak{d}_{\omega, \lambda}^{\omega}$ since $\operatorname{cof}(J \mid A) \leq \operatorname{cof}(J)$.

6. $J^{+} \stackrel{\omega}{\prec}\left(J^{+}\right)^{\omega}$. For $a, b \in P_{\omega}(\lambda)$, we let $a \prec b$ whenever $a \subseteq b$ and $\max (a \cap \omega)<\max (b \cap \omega)$.

Given an ideal $J$ on $P_{\omega}(\lambda), J^{+} \underset{\prec}{\stackrel{\omega}{\gtrless}}\left(J^{+}\right)^{2}$ means that for all $A \in J^{+}$and $F: \omega \times P_{\omega}(\lambda) \rightarrow 2$, there is $B \in J^{+}$such that $F$ is constant on

$$
\{(\max (a \cap \omega), b) \in \omega \times B: a \in B \text { and } a \prec b\} .
$$

This partition property, which is studied in [11] and [9], is clearly weaker than the property $J^{+} \stackrel{\omega}{\rightarrow}\left(J^{+}\right)^{2}$ considered above. The following shows that if $\operatorname{cof}(J)<\operatorname{cov}(M)$, then $J^{+} \stackrel{\omega}{\longrightarrow}\left(J^{+}\right)^{2}$ and $J^{+} \underset{\prec}{\stackrel{\omega}{\prec}}\left(J^{+}\right)^{2}$ are equivalent in a strong sense.

Proposition 6.1. Let $J$ be a fine ideal on $P_{\omega}(\lambda)$ with $\operatorname{cof}(J)<\operatorname{cov}(M)$, and let $A \in J^{+}$. Then there is $C \in J^{+} \cap P(A)$ with the property that for all $a, b \in C$ with $\max (a \cap \omega)<\max (b \cap \omega)$, one can find $c \in C$ with $c \prec b$ and $\max (c \cap \omega)=\max (a \cap \omega)$.

Proof. Set $A_{n}=\{a \in A: \max (a \cap \omega)=n\}$ for each $n \in \omega$. Define $c_{n} \in$ $P_{\omega}(\lambda)$ for $n \in \omega$ as follows. If $A_{0} \neq \emptyset$, let $c_{0}$ be an arbitrary member of $A_{0}$. Otherwise let $c_{0}=\{0\}$. Suppose $c_{0}, \ldots, c_{m}$ have already been constructed. If $A_{m+1}=\emptyset$, put $c_{m+1}=\{0, \ldots, m+1\}$. If $A_{m+1} \neq \emptyset$ and $A_{m+1} \cap \widehat{c}_{0}=\emptyset$, let $a_{m+1}$ be an arbitrary member of $A_{m+1}$. Finally if $A_{m+1} \cap \widehat{c}_{0} \neq \emptyset$, let $c_{m+1}$ be an arbitrary member of $A_{m+1} \cap \widehat{\bigcup_{i \leq r}} c_{i}$, where $r$ is the greatest $j \leq m$ such that $A_{m+1} \cap \widehat{\bigcup_{i \leq j}} c_{i} \neq \emptyset$.

Select $B_{\alpha} \in J$ for $\alpha<\operatorname{cof}(J)$ so that $J=\bigcup_{\alpha<\operatorname{cof}(J)} P\left(B_{\alpha}\right)$. For $\alpha<$ $\operatorname{cof}(J)$ and $n \in \omega$, let $D_{\alpha}^{n}$ be the set of all $s \in 2^{n+1}$ such that $s(n)=1$ and there is $b \in A_{n}-B_{\alpha}$ with the property that $c_{m} \subseteq b$ whenever $m$ is less than or equal to some $i<n$ with $s(i)=1$. Given $\alpha<\operatorname{cof}(J)$, let $D_{\alpha}=\bigcup_{n \in \omega} D_{\alpha}^{n}$ and $U_{\alpha}=\bigcup_{s \in D_{\alpha}} O_{s}$.

Let us prove that the open set $U_{\alpha}$ is dense. Thus let $k \in \omega$ and $p \in 2^{k}$. Pick $b \in A-B_{\alpha}$ so that $\bigcup_{m<k} c_{m} \subseteq b$ and $\max (b \cap \omega) \geq k$. Define $q \supset p$ by $\operatorname{dom}(q)=\max (b \cap \omega)+1, q(\max (b \cap \omega))=1$ and $q(i)=0$ for all $i$ with $k \leq i<\max (b \cap \omega)$. Then $q \in D_{\alpha}^{\max (b \cap \omega)}$. 
Select $f \in \bigcap_{\alpha<\operatorname{cof}(J)} U_{\alpha}$. For each $\alpha<\operatorname{cof}(J)$, pick $s_{\alpha} \in D_{\alpha}$ with $s_{\alpha} \subset f$.
Put

$$
Y=\left\{\max \left(\operatorname{dom}\left(s_{\alpha}\right)\right): \alpha<\operatorname{cof}(J)\right\}
$$

and let $m_{0}, m_{1}, \ldots$ be the increasing enumeration of the elements of $Y$. Set $E_{0}=A_{m_{0}}$ and for each $l \in \omega$,

$$
E_{l+1}=\left\{a \in A_{m_{l+1}}: \bigcup_{i \leq l} c_{m_{i}} \subseteq a\right\} .
$$

Finally set $C=\bigcup_{l \in \omega} E_{l}$. If $\alpha<\operatorname{cof}(J)$ and $l \in \omega$ are such that $\max \left(\operatorname{dom}\left(s_{\alpha}\right)\right)=m_{l}$, then $s_{\alpha} \in D_{\alpha}^{m_{l}}$ and $s_{\alpha}\left(m_{i}\right)=1$ for all $i<l$, and therefore there is $b \in A_{m_{l}}-B_{\alpha}$ such that $\bigcup_{j \leq m_{i}} c_{j} \subseteq b$ for all $i<l$. It clearly follows that $C \in J^{+}$and $c_{m_{l}} \in E_{l}$ for all $l \in \omega$. Finally given $d, e \in C$ with $\max (d \cap \omega)<\max (e \cap \omega)$, we have $c_{\max (d \cap \omega)} \subseteq e$.

\section{References}

[1] T. Bartoszyński and H. Judah, Measure and category - filters on $\omega$, in: Set Theory of the Continuum, H. Judah et al. (eds.), Math. Sci. Res. Publ. 26, Springer, New York, 1992, 175-201.

[2] J. E. Baumgartner, Remarks on partition ordinals, in: Set Theory and its Applications, J. Steprāns and S. Watson (eds.), Lecture Notes in Math. 1401, Springer, Berlin, 1989, 5-17.

[3] J. E. Baumgartner and A. D. Taylor, Partition theorems and ultrafilters, Trans. Amer. Math. Soc. 241 (1978), 283-309.

[4] J. E. Baumgartner, A. D. Taylor and S. Wagon, Structural properties of ideals, Dissertationes Math. 197 (1982).

[5] A. Blass, Combinatorial cardinal characteristics of the continuum, in: Handbook of Set Theory, M. Foreman et al. (eds.), Kluwer, Dordrecht, to appear.

[6] R. M. Canjar, On the generic existence of special ultrafilters, Proc. Amer. Math. Soc. 110 (1990), 233-241.

[7] J. Ketonen, On the existence of P-points in the Stone-Čech compactification of integers, Fund. Math. 92 (1976), 91-94.

[8] P. Matet, A partition property of a mixed type for $P_{\kappa}(\lambda)$, preprint.

[9] - Negative partition relations for uncountable cardinals of cofinality $\omega$, Studia Sci. Math. Hungar. 37 (2001), 233-236.

[10] P. Matet and J. Pawlikowski, Ideals over $\omega$ and cardinal invariants of the continuum, J. Symbolic Logic 63 (1998), 1040-1054.

[11] P. Matet and C. Péan, Distributivity properties on $P_{\omega}(\lambda)$, preprint.

[12] P. Matet, C. Péan and S. Shelah, Cofinality of normal ideals on $P_{\kappa}(\lambda)$, preprint.

[13] F. P. Ramsey, On a problem of formal logic, Proc. London Math. Soc. 30 (1930), 264-286.

[14] M. Scheepers, The least cardinal for which the Baire category theorem fails, Proc. Amer. Math. Soc. 125 (1997), 579-585. 
[15] J. E. Vaughan, Small uncountable cardinals and topology, in: Open Problems in Topology, J. van Mill and G. Reed (eds.), North-Holland, Amsterdam, 1990, 195218.

Université de Caen-CNRS

ESA 6081

Laboratoire SDAD

Campus II

14032 Caen Cedex, France

E-mail: matet@math.unicaen.fr

Received 20 July 2000;

in revised form 14 June 2001 